\title{
THE RELATION BETWEEN LEWIS'S STRICT IMPLICATION AND BOOLEAN ALGEBRA $\dagger$
}

BY E. V. HUNTINGTON

1. Introduction. The purpose of this note is to point out that the relation called "strict implication" in C. I. Lewis's system of logic can be shown to be substantially equivalent to the relation called subsumption $(p<q)$ in ordinary Boolean algebra.

The proof hinges upon the establishment of two new theorems, numbered 23.1 and 23.2 below.

2. Notation. The principal symbols which occur in the formulas of Lewis's system are the following. [For convenience of printing, we shall use,+ ', and * in place of Lewis's "wedge" $(\vee)$, "curl" $(\sim)$, and "curl-diamond" $(\sim \diamond)$; and to avoid confusion between Lewis's double use of the $\operatorname{sign}=$, we shall replace one of these signs by $\sim$.]

(1). $p, q, r$, etc. are variables, elements of an undefined class $K$ (interpretable as propositions).

(2). $p \times q$, or simply $p q$ (read: $p$ times $q$ ), is an object determined in an undefined way by the two elements $p$ and $q$, and called their logical product.

(3). $p+q$ (read: $p$ plus $q$ ) is an object determined in an undefined way by the two elements $p$ and $q$, and called their logical sum.

(4). $p^{\prime}$ (read: $p$ prime) is an object determined in an undefined way by the element $p$, and called the contradictory of $p$.

(5). $p-3 q$ (which may be read: $p$ hook $q$ ) is also an object determined in an undefined way by the elements $p$ and $q$. It may be called the "implication of $p$ toward $q . "$

(6). $p^{*}$ (which may be read: $p$ star) is an object determined in an undefined way by the element $p$. For lack of a better name, $p^{*}$ may be called the "ghost of $p$."

(7). $p \sim q$ (which may be read: $p$ wave $q$ ) is an object determined in an undefined way by the elements $p$ and $q$. It may be called the "equalization of $p$ and $q . "$

Finally, the elements of the class $K$ are classified into those

$\dagger$ Presented to the Society, June 20, 1934. 
which are "accepted" in the system, and those that are not. To distinguish between these two classes, it will be convenient to introduce the following notation, which serves the same purpose as the Frege assertion sign. $\dagger$

(8). " $p$ in $T$ " (read: $p$ is in $T)$, is an abbreviation for the assertion " $p$ is an accepted element in the system."

On the basis of this notation, three relations may be defined as follows:

(9). " $p$ implies $q$ " is merely another way of saying " $(p-3 q)$ is in $T . "$

(10). " $p$ is impossible," or " $p$ is a ghost," is merely another way of saying " $p$ * is in $T$."

(11). $p=q$ (read: " $p$ is equal to $q$ ") is merely another way of saying " $(p \sim q)$ is in $T$."

3. Rules of Procedure. In every abstract deductive system, later formulas are derived from earlier ones in accordance with certain rules of procedure.

The rules of procedure adopted in Lewis's system are the following:

I. Rule of inference. Whenever in the course of the development of the system we find established the expression " $p$ in $T$," and also the expression " $p-3 q$ in $T$," we may thereupon write down the expression " $q$ in $T$." More briefly:

$$
[(p \text { in } T) \text { and }(p-3 q \text { in } T)] \rightarrow(q \text { in } T),
$$

where the "arrow" notation may be read: the antecedent "leads to" the consequent. Obviously, whenever $x \rightarrow y$ and $y \rightarrow z$, then $x \rightarrow z$.

II. Rule of "replacement throughout." If we find that an expression " $F(p, q, r, \cdots)$ in $T$ " has been established, then we may replace $p$ by any other element "throughout the expression" (that is, we must make the replacement simultaneously at every point where $p$ occurs) ; similarly, we may replace $q$ by any other element "throughout the expression"; etc. Here $F(p, q, r, \cdots)$

$\dagger$ This use of a "subclass $T$ " was suggested by the present writer in 1933 . See Transactions of this Society, vol. 35 (1933), p. 291, a nd this Bulletin, vol. 40 (1934), p. 127. 
is any expression built up out of $p, q, r$, etc. by means of the operators of the system.

III. Rule of "replacement by equals." If we find that the expression " $p=q$ " has been established, then at any point where $p$ occurs in any formula of the system, we may replace $p$ by $q$.

These two rules, taken together, are often called the rules of substitution.

IV. Rule of "adjunction".

$[(p$ in $T)$ and $(q$ in $T)] \rightarrow(p q$ in $T)$.

V. Rules of equivalence.

$$
[(p-3 q)(q-3 p) \text { in } T] \rightarrow(p=q) ; \text { and }
$$

$$
(p=q) \rightarrow[(p-3 q)(q-3 p) \text { in } T] \text {. }
$$

(c)

$$
[(p-3 q) \text { in } T] \text { and }[(q-3 p) \text { in } T] \rightarrow(p=q) \text {. }
$$

Here (c) follows from (a) by aid of the rule of adjunction.

VI. Finally, we are to understand that whenever $p$ and $q$ are elements of $K$, the objects denoted by $p q, p+q, p^{\prime}, p-3 q, p^{*}$ and $p \sim q$ are also elements of $K$.

4. Established Theorems. Starting from certain primitive propositions, or postulates, each of which is an expression of the form " $F(p, q, r, \cdots)$ in $T$," and employing the rules of procedure, Lewis establishes, among others, the following important theorems. [The references are to Symbolic Logic by Lewis and Langford (The Century Co., 1932).]

1. If $p$ and $q$ are in $K$, then $p q$ is in $K$.

2. If $p$ is in $K$, then $p^{\prime}$ is in $K$.

3. $p q=q p$.

4. $(p q) r=p(q r)$.

Def. $p+q=\left(p^{\prime} q^{\prime}\right)^{\prime}$.

[Page 123]

5. $p q+p q^{\prime}=p$.

6. $p-3 p$ in $T$.

7. $(p-3 q)-3\left(q^{\prime}-3 p^{\prime}\right)$ in $T$.

8. $\left(p-3 p^{\prime}\right)-3(p-3 q)$ in $T$.

[18.12 with 19.74]

9. $(p-3 q)=\left(p q^{\prime}\right)^{*}$.

10. $p^{*}=p-3 p^{\prime}$. 
The following theorems 11-20 are deducible from 1-5 alone, since (by Rule III) 1-5 make the system a Boolean algebra. $\dagger$

11. $p+q=\left(p^{\prime} q^{\prime}\right)^{\prime}$.

12. $p(q+r)=p q+p r$.

13. $p^{\prime \prime}=p$.

14. $p=p p$.

15. $p p^{\prime}=q q^{\prime}$.

Proof. By 13, 11, 5, 3, 4,

and

$$
\begin{aligned}
\left(p p^{\prime}\right)^{\prime} & =\left(p^{\prime \prime} p^{\prime}\right)^{\prime}=p^{\prime}+p=\left(p^{\prime} q+p^{\prime} q^{\prime}\right)+\left(p q+p q^{\prime}\right) \\
& =q^{\prime} p+q^{\prime} p^{\prime}+q p+q p^{\prime},
\end{aligned}
$$

$$
\left(q q^{\prime}\right)^{\prime}=\left(q^{\prime} p+q^{\prime} p^{\prime}\right)+\left(q p+q p^{\prime}\right) .
$$

Hence $p p^{\prime}=q q^{\prime}$, by 13 .

16. Def. $Z=p p^{\prime}$.

17. $p Z=Z$.

Proof. By 16, 4, 14, 16, $p Z=p\left(p p^{\prime}\right)=(p p) p^{\prime}=p p^{\prime}=Z$.

18. $Z+p=p$.

Proof. By 5, 14, 16 (and 11,3), $p=p p+p p^{\prime}=p+Z=Z+p$.

19. $Z=(p q) p^{\prime}$.

Proof. By 17, 16, 4, 3, $Z=q Z=q\left(p p^{\prime}\right)=(q p) q^{\prime}=(p q) p^{\prime}$.

20. $p q^{\prime}=p(p q)^{\prime}$.

Proof. By 18, 16, 12, 11, 13,

$p q^{\prime}=Z+p q^{\prime}=p p^{\prime}+p q^{\prime}=p\left(p^{\prime}+q^{\prime}\right)=p\left(p^{\prime \prime} q^{\prime \prime}\right)^{\prime}=p(p q)^{\prime}$.

From 9 , by the aid of 19 , we have also the interesting formula

21. $Z^{*}=(p q-3 p)$.

Proof. By $19, Z^{*}=\left[(p q) p^{\prime}\right]^{*}$, and by $9,\left[(p q) p^{\prime}\right]^{*}=p q-3 p$.

5. New Theorems. The following formulas, which are not explicitly mentioned in Lewis's book, are directly deducible from propositions 1-10. (The proof will be given below.)

23.1 .

$$
(p-3 q)-3[(p-3 p q)(p q-3 p)] \text { in } T \text {; and }
$$

23.2 .

$$
[(p-3 p q)(p q-3 p)]-3(p-3 q) \text { in } T \text {; }
$$

whence, by $\mathrm{V}(\mathrm{c})$ with II,

$$
\text { 23.3. } \quad(p-3 q)=[(p-3 p q)(p q-3 p)] \text {. }
$$

† See Transactions of this Society, vol. 35 (1933), pp. 280-286, 557; or Mind, vol. 42 (1933), pp. 203-207. 
Then from 23.3, by III,

$$
[(p-3 q) \text { in } T] \rightarrow\{[(p-3 p q)(p q-3 p)] \text { in } T\} .
$$

But by V(a), $[(p-3 p q)(p q-3 p)$ in $T] \rightarrow(p=p q)$. Hence

$$
\text { 24.1. }[(p-3 q) \text { in } T] \rightarrow(p=p q) \text {. }
$$

Again, from 23.3, by III,

$$
\{[(p-3 p q)(p q-3 p)] \text { in } T\} \rightarrow[(p-3 q) \text { in } T] .
$$

But by $\mathrm{V}(\mathrm{b}),(p=p q) \rightarrow[(p-3 p q)(p q-3 p)$ in $T]$. Hence

24.2. $(p=p q) \rightarrow[(p-3 q)$ in $T]$.

These theorems 24.1 and 24.2 give us our main result as follows:

24.3. Whenever we find the formula " $p-3 q$ " asserted, we may thereupon write down the formula " $p=p q$ "; and conversely, whenever we find the formula " $p=p q$ " established, we may write down that the formula " $p-3 q$ " is asserted.

Now in Boolean algebra the relation of subsumption $(p<q$, read " $p$ within $q$ ") is defined by the formulas

$$
\text { 25.1. }(p<q) \rightarrow(p=p q) \text {, and }(p=p q) \rightarrow(p<q) \text {; }
$$

hence (in view of Rule III) 24.3 may be written as

$$
\text { 25.2. }(p<q) \rightleftarrows[(p-3 q) \text { in } T] \text {. }
$$

Here $p<q$ is the Boolean relation of inclusion, and the "mutual arrow" notation, $x \rightleftarrows y$, means that each side "leads to" the other (that is, whenever we find $x$ established, we may write down $y$, and whenever we find $y$ established, we may write down $x$ ).

Finally, if the words " $p$ implies $q$ " are understood strictly in the sense defined in (9) [under "Notation" above] and if the words " $p$ within $q$ " are understood strictly in the sense defined in 25.1 , we may write

25.3. ( $p$ implies $q) \rightleftarrows(p$ within $q)$,

where the "mutual arrow" denotes inter-deducibility.

The significance of this result lies in the fact that the relation $p<q$ can be defined, and all its properties deduced, on the basis of the simple postulates for Boolean algebra (theorems 1-5 
above), which involve only the familiar $K,+, \times,{ }^{\prime}$, without any reference to $p-3 q$ or $p^{*}$; so that the elements $p-3 q$ and $p^{*}$ become in large measure superfluous.

It is true that the elements $p-3 q$ and $p^{*}$ may be interpreted arbitrarily in a variety of ways (without violating the postulates of the system), as shown by the examples in Symbolic Logic, Appendix II; and from a purely mathematical point of view these examples have decided interest. But from the point of view of practical logic, the statement " $p-3 q$ is asserted" and the statement " $p *$ is asserted" would seem to be more important than the bare elements $p-3 q$ and $p^{*}$.

The point is that, in Lewis's system, no matter how the element $p-3 q$ may be interpreted, the statement " $p-3 q$ is asserted" is always equivalent to the statement " $p$ is within $q$ " $(p<q)$; and no matter how the element $p^{*}$ may be interpreted, the statement " $p *$ is asserted" is always equivalent to the statement " $p=$ the zero element, $p p^{\prime}$, of the system."

In passing, one important feature of Lewis's system (not explicitly mentioned in his book) should be pointed out, namely, the fact that the "subclass $T$ " may be chosen to a large extent arbitrarily. In fact, if $K$ is finite, $T$ may be a single element, or any power of 2 up to half of the whole class $K$. $\dagger$

6. Proofs of 23.1 and 23.2. We now supply the proofs omitted above.

23.1. $(p-3 q)-3[(p-3 p q)(p q-3 p)]$ in $T$.

Proof. By $10,9,8, p^{*}-3\left(p q^{\prime}\right) *$ in $T$.

By $7,\left[p^{*}-3\left(p q^{\prime}\right)^{*}\right]-3\left[\left(p q^{\prime}\right)^{* \prime}-3 p^{* \prime}\right]$ in $T$.

Hence by I, $\left(p q^{\prime}\right)^{* \prime}-3 p^{* \prime}$ in $T$.

Hence by II, $\left[\left(p q^{\prime}\right)\left(p q^{\prime}\right)^{\prime}\right]^{* \prime}-3\left(p q^{\prime}\right)^{* \prime}$ in $T$, whence by $16, Z^{* \prime}-3\left(p q^{\prime}\right)^{* \prime}$ in $T$.

But by $7,13,\left[Z^{* \prime}-3\left(p q^{\prime}\right)^{* \prime}\right]-3\left[\left(p q^{\prime}\right)^{*}-3 Z^{*}\right]$ in $T$.

Hence by I, $\left(p q^{\prime}\right)^{*}-3 Z^{*}$ in $T$.

Hence by $9,\left\{\left(p q^{\prime}\right)^{*} Z^{* \prime}\right\} *$ in $T$.

Hence by 20 , replacing $p$ by $\left(p q^{\prime}\right)^{*}$ and $q$ by $Z^{*}$, and using III, $\left\{\left(p q^{\prime}\right) *\left[\left(p q^{\prime}\right)^{*} Z^{*}\right]^{\prime}\right\} *$ in $T$.

† Compare Mind, vol. 43 (1934), pp. 181-198, where, if the optional postulate 13 is omitted, the subclass $A$ may be interpreted as a partial analog of the subclass $T$ of the present paper. 
Hence by $9,\left(p q^{\prime}\right)^{*}-3\left[\left(p q^{\prime}\right)^{*} Z^{*}\right]$ in $T$.

Hence by 20 and 19, $\left(p q^{\prime}\right)^{*}-3\left\{\left[p(p q)^{\prime}\right]^{*}\left[(p q) p^{\prime}\right]^{*}\right\}$ in $T$.

Hence by $9,(p-3 q)-3[(p-3 p q)(p q-3 p)]$ in $T$.

23.2. $[(p-3 p q)(p q-3 p)]-3(p-3 q)$ in $T$.

Proof. By 9 and $6,\left(p p^{\prime}\right)^{*}$ in $T$, whence by $16, Z^{*}$ in $T$.

Hence by 19 , when we replace $p$ by $\left(p q^{\prime}\right)^{*}$ and $q$ by $Z^{*}$, $\left\{\left[\left(p q^{\prime}\right)^{*} Z^{*}\right]\left(p q^{\prime}\right)^{* \prime}\right\} *$ in $T$.

Hence by $9,\left[\left(p q^{\prime}\right)^{*} Z^{*}\right]-3\left(p q^{\prime}\right)^{*}$ in $T$.

Hence by 20 and 19, $\left\{\left[p(p q)^{\prime}\right]^{*}\left[(p q) p^{\prime}\right]^{*}\right\}-3\left(p q^{\prime}\right)^{*}$ in $T$.

Hence by $9,[(p-3 p q)(p q-3 p)]-3(p-3 q)$ in $T$.

It will be noted that these proofs could be written out without the use of the "star" notation, since $p^{*}$ serves merely as an abbreviation for $p-3 p^{\prime}$, and $\left(p q^{\prime}\right)^{*}$ as an abbreviation for $p-3 q$.

HARVARD UNIVERSITY

ON INTEGRAL INVARIANTS OF NON-HOLONOMIC DYNAMICAL SYSTEMS†

BY A. E. TAYLOR

1. Introduction. It is well known that there are certain integral invariants associated with holonomic dynamical systems. Cartan $\ddagger$ demonstrated that it is possible to characterize a Hamiltonian system by means of the relative integral invariant

$$
\int_{C} \sum_{i} p_{i} d q_{i}-H d t
$$

The purpose of this paper is to extend the theory to the case of non-holonomic systems.

We shall adopt the following conventions in notation. There are three ranges of indices, which we shall usually represent by

$\dagger$ Presented to the Society, June, 20, 1934. I wish to acknowledge my indebtedness to A. D. Michal for criticism and suggestions during the writing of this paper.

$\ddagger$ E. Cartan, Leçons sur les Invariants Intégraux, 1922, p. 13. Also W. F. Osgood, this Bulletin, vol. 39 (1933), p. 882, Abstract No. 343. 